\title{
A performance Evaluation of Shape and Texture based methods for Vein Recognition
}

\author{
*Zhongli Wang, **Baochang Zhang, Weiping Chen, Yongsheng Gao \\ *Computer Science and Engineering, Beijing Institute of Technology,P.R.China \\ **Institute for Integrated and Intelligent System, Griffith University, Australia \\ zlwang@mae.cuhk.edu.hk,bczhang@jdl.ac.cn
}

\begin{abstract}
This paper gives fair comparisons of shape and texture based methods for vein recognition. The shape of the back of hand contains information that is capable of authenticating the identity of an individual. In this paper, two kinds of shape matching method are used, which are based on Hausdorff distance and Line Edge Mapping(LEM) methods. The vein image also contains valuable texture information, and Gabor wavelet is exploited to extract the discriminative feature. In order to evaluate the system performance, a dataset of 100 persons of different ages above 16 and of different gender, each has 5 images per person is used. Experimental results show that Hausdorff, LEM and Gabor based methods achieved 58\%, 66\%, 80\% individually.
\end{abstract}

\section{Introduction}

Biometric is the science of identifying a person using the physiological or behavioral features. Recently, vein pattern has attracted increasing attention. The physical shape of the subcutaneous vascular tree of the back of the hand contains information that is capable of authenticating the identity of an individual[1,2]. A vein pattern is the vast network of blood vessels underneath a person's skin. It is like fingerprint, which is widely used in person identification. However, compared with fingerprint, vein patterns are mostly invisible to human eye, therefore, they are more stable biometric features for person identity verification.

A typical vein pattern biometric system consists of five individual processing stages: image acquisition, image enhancement, vein pattern segmentation, feature extraction and matching. The database we used is from University of Tennessee, one can refer to[2].As for the similarity between vein and fingerprint, the researchers can get good results by using fingerprint recognition method, such as Gabor based method [5]. In this paper, shape and texture based methods are exploited for the performance evaluation. For shape based methods, Hausdoff and LEM are used as the distance measure, while for the texture based method, Gabor magnitude feature is combined with nearest neighbour classifier using the Euclidian similarity measure.

The rest part of paper is organized as follows. In section 2, image enhancement and feature matching methods for the shape based vein recognition are briefly introduced. In section 3, Gabor based method is proposed for vein recognition. In section 4 , comparative experiment is conducted on a database of 500 image for 100 persons. Conclusion and future work appear in section 5 .

\section{Image enhancement and shape matching for vein recognition}

The key step for shape based vein recognition is binary preprocessing for the grey-level image. As for the vein vary across the image, global thresholding techniques alone do not provide satisfactory results. This paper chooses different threshold value for every pixel in the image based on the local neighbors.

\subsection{Binary image for vein pattern}

The quality of the vein image is not well for global binary method because the vein database was acquired using the low-cost device. Due to the fact that the graylevel intensity values of the vein vary slowly across the image, global thresholding techniques alone do not provide the satisfactory results. To obtain a better representation of the shape of the vein pattern, we mainly used local thresh holding method based on local mean value based on a given threshold $T_{g}$.

$$
\mu_{i, j}=\frac{1}{M . N} \sum_{i}^{M} \sum_{j}^{N} I(i, j)
$$


where $M$ and $N$ are indices of local rectangular region.

$$
I(i, j)=\left\{\begin{array}{l}
1, \text { if } I(i, j)>=\left(\mu_{i, j}-T_{g}\right) \\
0, \text { otherwise }
\end{array}\right.
$$

In this paper, $T_{g}=5$ is used as the common offset for all pixels, which are chosen on trial-and-error basis, and local region is set as $13 \times 13$. One sample in Fig. 1 shows the vein pattern has been successfully segmented from the original image after applying the local thresholding and thinning algorithms.

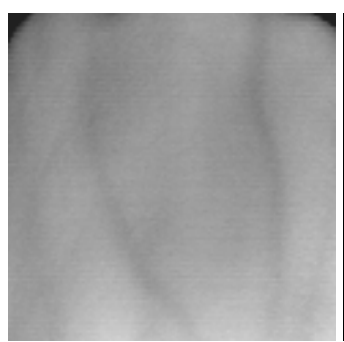

a)

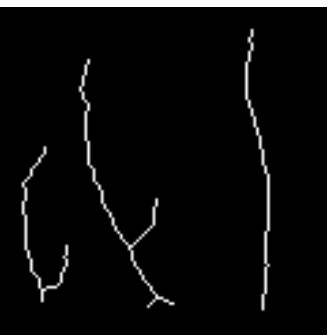

b)
Figure 1. a) is original vein image, b) is binary image for a).

\subsection{Hausdorff based vein recognition}

In this section, the hausdorff based shape matching method is briefly described. The hausdorff distance is defined as follows:

$$
h(A, B)=\max _{a \in A}\{\min \{d(a, b)\}\}
$$

where $\mathrm{a}$ and $\mathrm{b}$ are points of sets $\mathrm{A}$ and $\mathrm{B}$ respectively, and $d(a, b)$ is any metric between these points; for simplicity, we will take $d(a, b)$ as the Euclidian distance between $a$ and $b$. After binary vein images are calculated using method in section 2, Eq. 3 is used for the identity classification.

Y. Gao, et al. proposed another kind of the hausdorff distance, the so-called Line Edge Map(LEM), for face recognition. This paper also uses it for vein recognition. Details about LEM can refer to [3].

\section{Gabor feature based vein recognition}

Gabor wavelet models quite well the receptive field profiles of cortical simple cells, therefore, Gabor feature can capture the salient visual properties such as the spatial localization, orientation selectivity, and spatial frequency characteristic[4,5]. The Gabor wavelets (kernels, filters) can be defined as follows:

$$
\psi_{u, v}(\mathbf{z})=\left(\left\|\mathbf{k}_{u, v}\right\|^{2} / \sigma^{2}\right) e^{\left(-\left\|\mathbf{k}_{u, v}\right\|^{2}\|\mathbf{z}\|^{2} / 2 \sigma^{2}\right)}\left[e^{i \mathbf{k}_{u, v} \cdot \mathbf{z}}-e^{-\sigma^{2} / 2}\right]
$$

where $\mathbf{k}_{u, v}=\left(\begin{array}{l}k_{v} \cos \Phi_{u} \\ k_{v} \sin \Phi_{u}\end{array}\right), k_{v}=\frac{f_{\max }}{2^{v+2}}, \Phi_{u}=u \frac{\pi}{8}, v$ is the scale(frequency), and $u$ is the orientation with $f_{\max }=\sqrt{2} \pi / 2$ and $\mathbf{z}=(x, y)^{T}$. We used 5 scales and 8 orientations for vein recognition. It is well-known that Gabor wavelets can be used to enhance the features in certain scales and orientations, and have been widely used in image processing and object recognition.

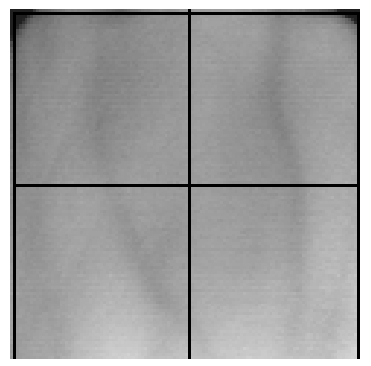

Figure 2. A example divided into 4 rectangular regions

Let $G_{u, v}(Z)$ denotes the Gabor magnitude feature of an image, where $u$ and $v$ are the orientation and scale of the kernel, respectively. The feature extraction is based on the multi-region (see Fig.2) mean Gabor magnitude feature, represented by $\overline{G \mu}_{u, v, l}$ as

$$
\overline{G \mu}_{u, v, l}=\frac{1}{N} \sum_{Z_{i} \in R_{l}} G_{u, v}\left(Z_{i}\right)
$$

where $l=1,2, \ldots, L$ is the region index, and $L$ is the number of sub-region. $N$ represents the total number of pixels in the region $R_{l}$. Details about the method can refer to [5]. In the classification phase, the nearest neighbor classifier is used with the Euclidian distance as the similarity measure. In the experiment, $L=64$.

\section{Experiments}

In this section, a database of 500 images for 100 persons is used to compare the performance of the shape and texture based methods. In the database, each person has 5 images, one is used as target set, others are in query set. Two persons' samples are shown in Fig.3. In the experiment, the vein images are normalized to the size of $100 \times 100$. 


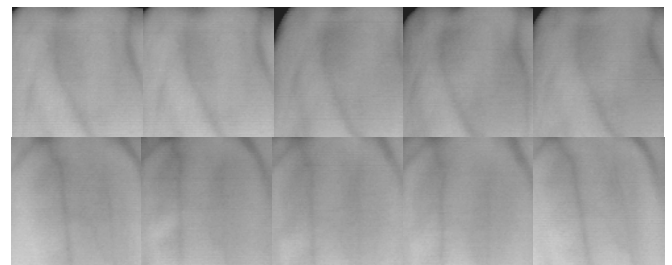

Figure 3. amples of Vein, the first row is labeled as 00001 , the second row is labeled as 00002 .

As shown in Table.1, Gabor feature get the best performance, and line edge map method achieves much better performance than point-based hausdorff method. It is interesting that Gabor feature can get much better performance than traditional shape based methods, the reason for which is that Gabor wavelet is robust to variation on gray-level images. In addition, region-based method can reserve the structure information, which is very important for vein recognition. We should mention that the normalized images are not well alignment, which are one of main problem for vein recognition system.

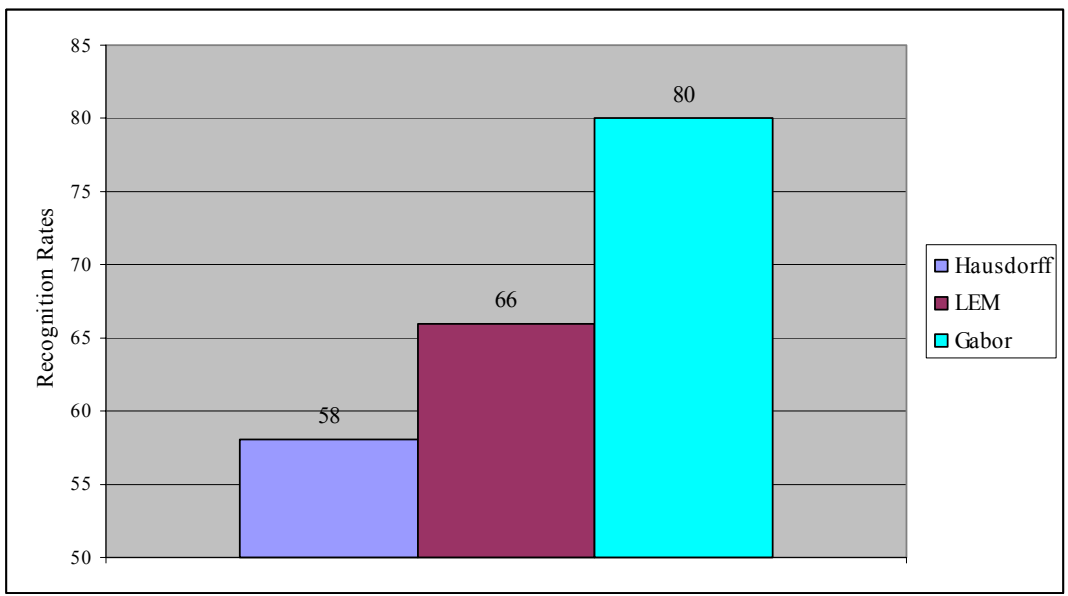

Fig. 4. Recognition rates for shape and texture based vein recognition methods

\section{Conclusion and future work}

This paper gives fair performance comparison for vein recognition, which is one of most secure biometric recognition methods. A database of 500 images for 100 persons is used to evaluate three methods, point-based hausdorff, line edge map, and Gabor based methods. The Gabor feature gets the best performance with $80 \%$ recognition rates, line edge map gets $66 \%$, which is much than point-based hausdorff based method. In the future work, we will make further investigation about the image enhancement and feature extraction.

\section{Acknowledgment}

We would like to express our sincere appreciation to Ahmed M. Badawi from University of Tennessee, who share the valuable database to us.

\section{References}

[1] Ahmed M. Badawi, "Hand Vein Biometric Verification Prototype: A Testing Performance and Patterns Similarity," In Proceedings of the International Conference on Image Processing, Computer Vision, and Pattern Recognition, 2006, pp.3-9

[2] Lingyu Wang, Graham Leedham, Siu-Yeung Cho, "Minutiae feature analysis for infrared hand vein pattern biometrics," Pattern Recognition, 2007.

[3] Y. Gao and M. Leung, "Face Recognition Using Line Edge Map," IEEE Transactions on Pattern Analysis and Machine Intelligence, Vol. 24, No.6, 2002, pp.764-809

[4] D. Gabor, "Theory of communication," Journal of the Institution of Electrical Engineers, vol. 93, part III, no. 26, 1946, pp. 429-457

[5] A. K. Jain, S. Prabhakar and L. Hong, "A Multichannel Approach to Fingerprint Classification", IEEE Transactions on Pattern Analysis and Machine Intelligence, Vol.21, No.4, 1999, pp. 348-359 